\title{
Assessment of carers' psychological needs
}

\section{Jan Oyebode}

\begin{abstract}
Carers play a vital role in supporting family members who need help. Facts and figures on caregiving are set in the context of current UK policy. Research into the impact of caregiving shows that one-third to one-half of carers suffer significant psychological distress. Factors associated with carer stress are reviewed, including those related to the care-recipient, the caregiver, their relationship, and wider family and social support. The most well-known frameworks for understanding caregiving stress, including stress-process models, are introduced. Measures that may help in assessing psychological needs of carers are reviewed, emphasising the distinction between objective and subjective stress. The range of possible interventions leads to promising evidence of the effectiveness of individual psychosocial interventions, but further research is needed.
\end{abstract}

There is no question that families of those with mental illness are affected by the situation of their relative. In many cases, family members not only provide practical help and personal care but also give emotional support in the often inevitable change in the nature of the underlying emotional relationship. Thus, the relationship's usual characteristics are altered either temporarily during an acute episode of illness or on a long-term basis in the case of chronic conditions, and the balance changes as the parties become the caregiver and the carereceiver.

Those who receive care are, necessarily, dependent on their carer. This makes them vulnerable to the nature and quality of the care provided by that particular person. For the carer, the demands often not only bring direct stresses but also have indirect effects. For example, taking on a caring role may reduce the time, energy and finance available to devote to the other demands of life and this can affect work, social life and other relationships.

Nevertheless, caregiving may also have its satisfactions. Providing care and support to a relative is not a static process since the needs of the care-receiver alter as his or her condition changes. There is continual change and adjustment in the caring relationship and the role of the carer. Where there is acute disturbance or where care is required on a long-term and intensive basis, many carers will need to let others step in to share or take over responsibilities. It is not surprising, therefore, that being a carer often raises difficult personal issues about duty and responsibility, adequacy and guilt.

Given the additional energy, time and work that are entailed in providing care, alongside the emotional demands and wider impact on support systems, it is easy to see why many carers find that the role has a significant impact on their well-being.

It has taken a long time for mental health services to pay due attention to carers' needs (Box 1 ). There has, perhaps, been ambivalence about recognising that an individual other than the referred patient is a legitimate recipient of service time and expertise. However, a growing body of research into the impact of caregiving over the past 20 years has led to improved understanding, offering a sound basis for individual assessment and intervention. Groups in the voluntary sector have raised awareness of issues affecting carers and there are also policy reports and directives that recommend or require that attention be given to carers' needs. It is therefore timely to review good practice in the assessment of carers.

The material here is drawn almost entirely from research into caring for an elderly population. The majority of the studies focus on care for those with dementia, but many of the issues are relevant to the wider range of those caring for people with mental illness or disability.

\section{Box 1 A familiar issue}

'Despite advances in hospital and community services, however, we are still faced with many carers who are not only under stress, but also suffer from psychiatric and physical illness which may go unnoticed or ignored. Until their needs are met, we cannot claim to have a civilised and effective health service.' MacLennan (1998: p. 652)

Jan Oyebode is a clinical psychologist specialising in work with older people. She is a senior lecturer and Director of the Clinical Psychology Doctorate Course at the University of Birmingham (Course Director, Doctorate in Clinical Psychology, School of Psychology, The University of Birmingham, Edgbaston, Birmingham B15 2TT, UK. E-mail: j.r.oyebode@bham.ac.uk) and also spends 1 day a week in clinical practice within South Birmingham Mental Health NHS Trust. Her particular interests are in psychological adaptation to late-life events, including dementia and bereavement. 


\section{Definitions}

Here, the terms 'carer' and 'caregiver' are used interchangeably to refer to the person who provides care to another who is dependent on him or her for help. Zarit \& Edwards (1999) define caregiving as 'interactions in which one family member is helping another on a regular basis with tasks that are necessary for independent living' (p. 334). They state that becoming a carer for an older person reflects a change: 'A relationship develops into caregiving when an older person becomes dependent on another's help to complete tasks and another family member provides or arranges for this assistance' (p. 334). In these terms, becoming a carer involves taking on practical supportive tasks, although to care for someone has obvious emotional connotations. It is the weaving together of these elements that can make caregiving such a challenge.

\section{Facts and figures}

In the 1995 General Household Survey, it was estimated that there are 5.7 million carers in Britain, of whom 1.7 million provide over 20 hours of care per week (Office of National Statistics, 1997). Of the 5.7 million, $60 \%$ report providing care for an individual with physical disability, $18 \%$ for someone affected by ageing, $15 \%$ for someone with both mental and physical health problems and $7 \%$ for a person with a mental health problem alone.

There are an estimated 125000 carers in Britain living with a relative who has severe mental illness (Department of Health, 1999b; http://www.doh. gov.uk). Of these, about 10000 are carers of school age providing support to a single parent with mental health problems (Department of Health, 1999a; http://www. doh.gov.uk). They also include the many adults who provide assistance to a child or adult with learning disability. Half of all carers in the UK look after someone over 75 years of age (Office of National Statistics, 1997), thus underlining the predominance of informal care for elderly people which has arisen as a result of the changing demographic profile in Europe and North America.

In the USA, an estimated 5 million people are providing care for their parents at any one time, almost matched by the number of spouses providing assistance for an elderly partner (Zarit \& Edwards, 1999).

In the UK, the cost of providing care for individuals with dementia alone has been estimated at $£ 6.1$ billion per annum (at 1998/1999 prices), of which $£ 2.8$ billion (including lost income) is carried by informal carers (Audit Commission, 2000; http://www.audit-commission.gov.uk). In the USA, informal carers are reported to provide over $85 \%$ of all caregiving days in the community (Zarit \& Edwards, 1999).

A significant minority of carers are themselves elderly. Where a dependent person has a spouse, care is most likely to be provided by that person, whether husband or wife, although husbands receive more additional help than wives (Enright, 1991). The 1995 General Household Survey (Office of National Statistics, 1997) found that $27 \%$ of carers in the UK were themselves over 65 years old. Aside from spouses, however, provision of informal care is a largely female role. Where there is a widowed parent with adult children, care is more likely to be provided by daughters even where there are also sons. Where there are only sons, care is more likely to be provided by daughters-in-law than by the sons themselves.

\section{The impact of providing care}

Satisfying aspects within their caring role are identified by $55 \%$ to over $90 \%$ of family carers (Nolan et al, 1996). Nevertheless, these are often out-weighed by stresses. A number of studies have found that over one-third of those providing care for a relative with dementia suffer from high levels of stress, depression or general psychological morbidity (see Schultz et al, 1995, for a comprehensive review). They report more daily hassles than non-caregivers of a comparable age. They also have poorer physical health on self-report measures and take more prescribed medication than age-matched samples. Difficulty in coping with caring plays a key part in influencing placements into institutional care.

\section{Policies}

Given that informal carers provide a service that would otherwise cost health and social services a huge amount of money, it is logical that government policies should aim to ensure that they receive adequate support to continue to fulfil a caring role without damage to their own health and well-being. A number of recent policy initiatives in the UK have addressed carers' needs and emphasised the responsibility of services towards carers. These are summarised in Box 2.

\section{Models for understanding carer reactions}

It has therefore been established that over one-third of carers have significant levels of stress or distress. 
However, they play a vital role in helping those with illness to continue to live in the community. The next issue that arises is how clinicians can make sense of this stress response in a way that may help towards a useful intervention. There are a number of useful models for understanding carer stress.

One way of understanding carers' reactions is to focus on what is known about the processes of stress and adaptation. Pearlin et al (1990) and Nolan et al (1996) both draw on stress-process models in their frameworks for understanding the impact of caregiving.

Pearlin et al (1990) have drawn up a framework that allows the demands and resources of the caregiver to be clearly identified. They distinguish four domains: background and contextual factors; stressors; mediators of stress; and outcomes. The stressors are themselves divided into three types: primary stressors, directly connected with providing care; secondary role strains such as those caused by the conflicting demands of caring, work and family; and secondary intra-psychic strains, including the impact of caring on self-esteem. Pearlin et al have developed an interview schedule to pick up the major factors within these domains. The framework can account for many influential factors, which can include demographic, cultural and life-history influences. Mediating factors include resources for coping with social, economic and internal stresses. The schedule provides a useful way of making sense of a carer's particular situation and may suggest areas for intervention. Overall, this model is reasonably comprehensive, easy to understand and clinically relevant. The model is shown in diagrammatic form in Fig. 1.

By contrast, Nolan et al (1996) have produced a descriptive, stage-based, longitudinal model of the caregiving process, based on findings from interviews with carers who were supporting people with varying degrees of dementia. The six stages of this model are: building on the past; recognising the need; taking it on; working through it; reaching the end; a new beginning. The basic stress-appraisal-coping process (recognition and appraisal of stress; appraisal of resources and ways of coping; taking action and evaluating action) takes place within each of the stages. This model is helpful in prompting the clinician to consider the dynamic 'career' of the caregiver and to tailor any intervention to the appropriate stage of the process.

A rather different model has been put forward by Hall (1990), who proposes that the carer is guided

\section{Box 2 Recent UK Government policy, strategy and guidelines on carers}

The Carers (Recognition and Services) Act, 1995

This gives carers the right to request an assessment of their own needs from social services, although without a guarantee that any needs identified would be met. The implementation of the Act in relation to carers of those with mental illness has been described as patchy.

Caring about Carers: A National Strategy for Carers (Department of Health, 1999a)

This presents a 'substantial new policy package about carers', which draws together current initiatives, examples of good practice and proposals for the future. There is a focus on three areas: information, support and care. 'Information' stresses the need for good information to be available to carers and about carers. 'Support' refers to the need for carers to be involved as partners in planning and providing services. 'Care' focuses on government measures that will address carers' own needs.

Standard 6 of the National Service Framework for Mental Health (Department of Health, 1999b)

People providing regular and substantial care within the Care Programme Approach must be assessed at least annually to assess their caring, physical and mental health needs. They must also have a written care plan developed in discussion with them.

Forget Me Not: Mental Health Services for Older People (Audit Commission, 2000)

This publication provides a national review with recommendations about how health and social services should be providing mental health services for older adults. Conclusions were drawn from a multifaceted, in-depth look at services across agencies at 12 sites within England and Wales, identifying variation, good practice and shortcomings in services. The section on carers reflects themes similar to those in the National Strategy but with a focus on service rather than policy, the need for good information and for emotional and practical support.

Standard 7 of the National Service Framework for Older People (Department of Health, 2001)

This states the right of access of older people with mental health problems to integrated NHS and council mental health services that ensure effective diagnosis, treatment and support. It also states that carers should be offered information, advice and practical help to support them in caring for an older person. 


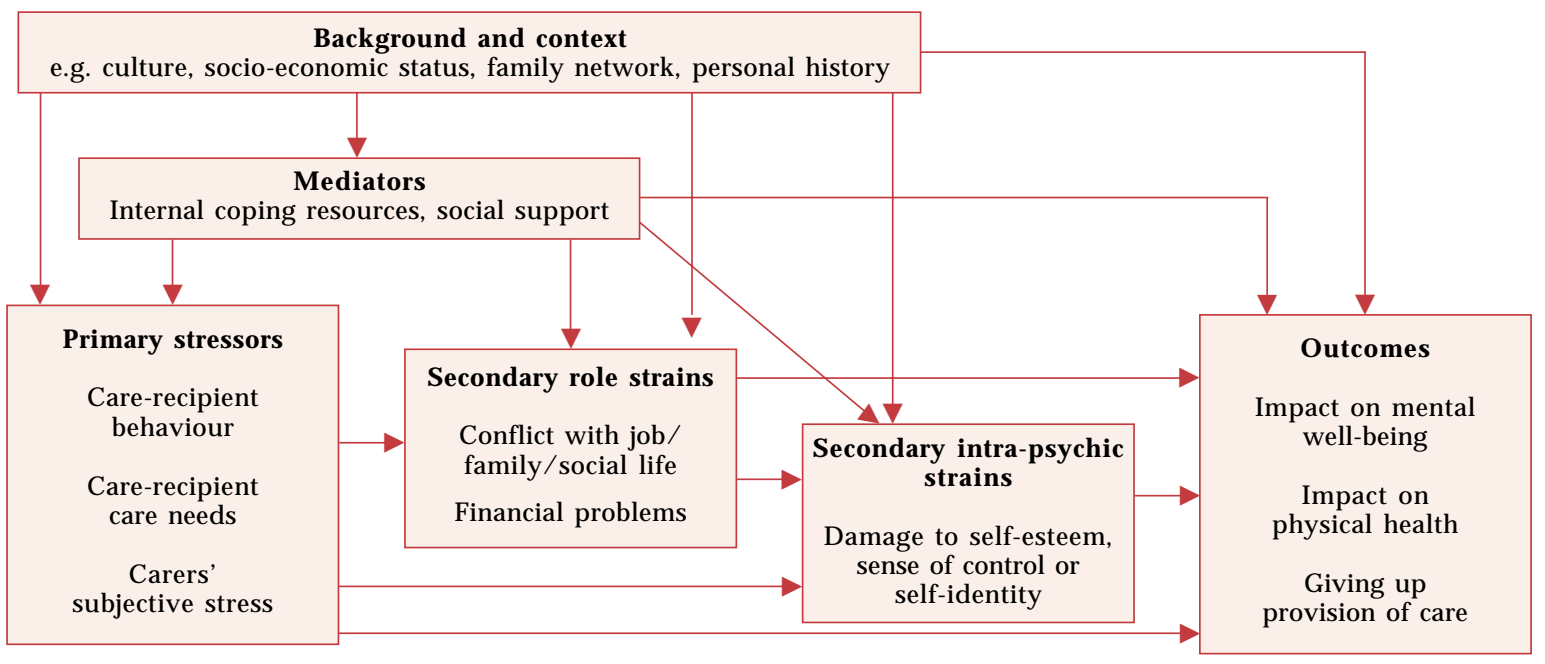

Fig. 1 Pearlin's stress-process model of stress in carers of those with Alzheimer's disease (adapted from Pearlin et al, 1990).

by an underlying, possibly unarticulated, set of beliefs. These, along with the care-recipient's needs, influence the carer's goals and objectives. The goals and objectives then determine a set of practices or acts which occur in the caring encounter and which are accompanied by emotions and feelings. The strengths of this model lie in its attention to the role of underlying beliefs and values and in its breadth of vision. These make it applicable to many caregiving situations and provide an alternative to the focus on stress and burden.

\section{Scales for measuring carer stress and coping}

In assessing the degree of carer stress, it is important to distinguish between objective and subjective burden. Objective burden is a measure of the additional practical support or activity that must be undertaken by the carer as a result of the care needs of the dependent person. Subjective burden consists of the emotional and cognitive reaction of the carer to the situation. The same degree of caregiving, in terms of tasks that must be done (i.e. objective burden), can be experienced very differently by carers (i.e. subjective burden), depending on their personal interpretation of their situation.

A number of measures have been devised to assist in assessment of carer stress. Some measure both objective and subjective burden and others focus only on the subjective dimension. The ones reviewed here have all been developed to assess stress among those caring for dependent older adults.

The Zarit Burden Interview (Zarit et al, 1980) is probably the most well-known. It is a 29 -item self-report scale which measures subjective burden. The sample used in the original study was very small, at 29 people, all of whom were providing care for a relative with memory problems. The range of possible answers from 'not at all' to 'extremely' is not clearly defined and the psychometric properties and construct validity of the scale are not clear. Nevertheless, its face validity is high, it is acceptable to carers and is widely used. In a thorough review of 53 articles on caring for an elder with cognitive impairment published between 1980 and 1997, Bedard et al (2000) found that the Zarit Burden Interview, or a measure based on it, was used in 25 $(47 \%)$ of the studies.

The Caregiving Burden Scale (Gerritsen \& Van der Ende, 1994) is a carefully constructed and validated scale which draws the majority of its items from the Zarit Burden Interview. It uses two factors to measure subjective burden: relationship and personal consequences. It is a 13-item scale that requires answers on a 5-point Likert scale from 'disagree very much' to 'agree very much'. The scale was developed in The Netherlands with a first sample of 89 carers for people with dementia and a second sample of 42 . It has sound psychometric properties and good construct validity. It is quick to administer and is acceptable to relatives, although some may require help to make distinctions within the 5-point response scale.

In contrast, the Caregiver Hassles Scale (Kinney \& Stephens, 1989) and the Behavioural Assessment Scale of Later Life (BASOLL; Brooker, 1998) both assess the degree of objective burden and the subjective level of stress that different aspects cause in the carer. Both scales are lengthy and detailed, giving a thorough picture of the support that is being 
provided as well as the carer's reactions to each aspect.

The BASOLL is primarily a behaviour scale and psychometric properties are not given for its component dealing with the reaction of the carer. Nevertheless, it gives a rich individual picture and can provide useful clinical information on where to target an intervention to relieve stress. The Caregiver Hassles Scale has good psychometric properties and the results distinguish stress in each of five domains such as hassle associated with assisting with the basic activities of daily living and hassle with the carer's support network. It does not tap stressors related to changes in the relationship per se.

It is useful not only to know what care individuals are providing and how this is affecting them emotionally but also to find out about their ways of coping. Nolan et al (1996) have developed a checklist, the Carers' Assessment of Managing Index (CAMI), which is useful in gaining an account from carers of the ways in which they cope and how useful these prove to be. It is helpful to pair it with the Carers' Assessment of Difficulties Index (CADI; Nolan \& Grant, 1992).

Matson (1994) has developed a Ways of Coping with Caring Scale that can be used to survey a carer's ways of managing. It provides three sub-scores that are linked with different coping styles.

\section{Factors influencing outcomes for carers}

Several factors influence how stressful, manageable or satisfying individual carers find their experiences. These are grouped below into those connected with the care-recipient, the caregiver and his or her relationship with the recipient, and the family context.

\section{Care-recipient factors \\ Severity of impairment}

It is sometimes assumed that the degree of stress experienced by a carer is directly related to the amount of instrumental care that needs to be provided. However, this is not necessarily the case. In a number of research surveys, no direct link has been found between degree of impairment or disability and the stress experienced by the carer (Coen et al, 1997).

\section{Care-recipient's behaviour}

Certain behaviour or features of the person requiring care have been linked with higher levels of stress in the caregiver. These include factors connected with high demands such as disturbed behaviour, restlessness, incontinence, emotional lability and night-time disturbance. Others are connected with withdrawal, including apathy, signs of depression in the care-recipient and decreased social activity. The latter reduce what has been called the mutuality of the relationship and may leave carers with little feeling of satisfaction. Where the care-recipient appears pleased, content or appreciative, carers report deriving much satisfaction from their role.

Thus, although an overall rating of level of impairment may not be useful in predicting carer strain, the presence in the care-recipient of disturbed or demanding behaviour or withdrawal may indicate that the caregiver is at risk of becoming stressed or depressed.

\section{Caregiver factors \\ Relationship to the care-recipient}

Many studies indicate differences between the type and degree of strain experienced by 'adult children' (the grown-up offspring of the care-recipient) and by spouses (Pruchno \& Resch, 1989; Baumgarten et al, 1992). These appear to arise from the caregiver's resources and demands, outside of the caregiving relationship. Thus, spouses, being older and usually dependent on a pension, may experience difficulty in coping with extra demand because of already lowered physical and financial resources. On the other hand, daughters often have responsibilities to other family members as well and may experience greater strain and role conflict. In shared households, caregivers report similar degrees of a decline in health, strains in relationships and restriction on activities, regardless of their relationship to the person being cared for.

Many spouses have a wholehearted commitment to giving care and report more areas of satisfaction than adult children. Their emotional investment may lead them to feel more involved, less burdened and less likely to seek institutional care than are adult children (Pruchno \& Resch, 1989). Daughters may report more distress than spouses. This is possibly because many spouses have already reduced the number of other commitments in their lives and may have expected to care for their partner, whereas daughters experience greater disruption to their lives to accommodate the caring role (Jones \& Vetter, 1984).

Overall, then, it may be less important to draw conclusions from whether the carer is a spouse or offspring than to be aware of the degree of emotional commitment of the caregiver and of the approximate balance of demands and resources in the major domains of life, such as physical, domestic, social and employment/financial aspects. 


\section{Adult children}

It is daughters rather than sons, at least in North American population samples, who most commonly feel a sense of obligation to provide care and take on the caregiving role. Caregiving daughters are likely to experience role strain, leading to Brody's description of them as 'women in the middle' (Brody, 1981). The degree of role conflict has been found to predict the degree of strain (Mui, 1992).

Intensive support is hard to fit in with work commitments and, as a consequence, daughter caregivers may give up work in order to look after their relative. The Government's National Strategy for Carers (Department of Health, 1999a) pays special attention to this issue, focusing on the need for carerfriendly employment practices and flexible working arrangements. Marriage has been found to buffer daughters from strain. Those who are married report receiving more support and suffering less from depression than those who have no partner (Brody et al, 1992). A comparison of co-resident (living with the parent) and non-resident (living separately from the parent) daughters found that the partnerships of those who lived apart from their parent were strained, whereas those who were co-resident found their activities restricted (Deimling et al, 1989).

Thus, caregiving daughters may be at risk for greater stress if they have conflicting roles and if they lack support from a partner.

\section{Gender of spouse}

Wives report greater strain than husbands but also report more behavioural and emotional problems in those for whom they care (Barusch \& Spaid, 1989). Clinicians therefore need to be aware of the occasions where there appears to be a lack of correspondence between the levels of reported problems and reported strain. They also need to be alert to the possibility that men may minimise the strain that they are under. One recent study found that husbands providing care were more anxious than wives (Croog et al, 2001), possibly because it is a less familiar role.

However, perhaps for the same reason, husbands may report satisfaction from the feeling that they can now repay their wives for all the care they have received from them in the past (Kaye \& Applegate, 1990). By contrast, wives, who may have spent a lifetime caring for children and elderly parents, may report resentment at finding themselves in a position where they need to take on this responsibility once again.

Men may respond to the challenges of giving care in a more task-oriented manner than women and with less emotional involvement (Zarit et al, 1986). They are also more likely to receive help from family and services. However, evidence suggests that, over time, the sense of subjective burden in wives may decrease to the same level found in husbands. This may be an indication that wives also learn to distance themselves emotionally and, like many men, become able to act in a manner more equivalent to a care manager than a personal care-provider.

\section{Intra-psychic factors}

The lack of correlation between objective impairment and level of carer stress suggests that cognitive, subjective interpretations influence carer well-being. The ability to find meaning in the situation enables many carers to continue and to draw satisfaction from caring. Research and current thinking would suggest that those carers who are able to draw on a range of coping strategies adapt more easily to the demands of caring. Nolan et al (1995) surveyed the range of coping strategies used by a sample of carers. They divided the possible strategies into 'managing events', 'managing meanings' and 'managing stress'. Some of the strategies most commonly used by carers in their survey are shown in Box 3 . There is some suggestion that a problem-solving, active coping style is associated with better well-being, whereas avoidance or emotion-focused coping results in poorer adaptation.Using factor analysis, Matson (1994) found three possible coping styles in his Ways of Coping with Caring questionnaire: 'active-involved', 'non-confronting' and 'value' coping. Of these, preference for a non-confrontational style, i.e. avoidance or taking the line of least resistance, was linked with stress and depression. Regardless of which coping style dominates, the most important aspect may be that the particular strategy employed fits the situation.

\section{Box 3 Coping strategies used by carers}

The nine most popular strategies, found to be very helpful by over $50 \%$ of carers, were:

- Realising that the person you care for is not to blame for his or her position

- Taking life 'one day at a time'

- Finding out as much as you can about the problem

- Keeping a little free time for yourself

- Realising that there is always someone worse off than yourself

- Realising that no one is to blame for things

- Keeping one step ahead of things by planning in advance

- Getting as much help as you can from professionals and service providers

- Talking over your problems with someone you can trust

(After Nolan et al, 1996) 
Low perceived control contributes directly to carer stress and depression. Carers may feel that they are controlled by their dependent relative or be afraid that services may take control. The latter can discourage stressed carers from accepting help such as day or respite care and is therefore an important issue of which those providing services should be aware.

\section{Quality of relationship}

Those with a poor premorbid relationship may experience more strain and those who are losing the intimacy of a previously close relationship may be at risk for depression. The style of the caregiver's attachment may also influence reactions to the situation.

The quality of the current relationship is a powerful factor influencing the feelings of the caregiver and whether continued care is given to his or her relative (Spruytte et al, 2001). The more positive the carer's rating of the current relationship, the less the degree of stress and depression experienced. Where there is a conflicting relationship, there may be a higher risk that the care-recipient will be physically abused (Reay \& Browne, 2001).

\section{Wider family aspects and social support}

The care function tends to fall on one person in the family, and adult children who are principal caregivers may feel angry towards, or abandoned by, siblings who are not greatly involved. Yet daughters who are not principal caregivers may feel more guilt than those who are providing care (Brody et al, 1989).

Whether contact with the wider family reduces caregiver stress may depend on the state of affairs within the family. If the wider family is experiencing a number of stressful events, carer stress is likely to be raised; conversely, where the wider family has more resources, caregiver stress is found to be lower. Social support has been shown to buffer against stress among carers of those with Alzheimer's disease. Interestingly, families may find themselves drawn closer together by a situation where a member becomes dependent, as they may share a common purpose and learn to appreciate the distinct contribution that each can make.

\section{Interventions}

The whole purpose of assessing a carer's situation is to gain an understanding that may help to prevent or to ameliorate stress-related problems, enabling the carer to continue providing care comfortably for their relative, assuming that this is what both would
Box 4 Factors that correlate with increased stress or poorer well-being in carers

Disturbed behaviour in the care-recipient Withdrawal in the care-recipient

Being a wife rather than a husband

Being a daughter rather than a spouse

Having conflicting roles and responsibilities

Having no support from a partner

Having no social support

Having a narrow range of coping strategies

Not using active, problem-solving coping strategies

Rating the current relationship as poor

Reporting no satisfactions from caring

wish. On occasion, the intervention may also appropriately lead to the carer letting the relative have care away from the home.

The basis of successful intervention lies in gaining an informed understanding of the individual case. Using models of caring may provide helpful ways of organising the information on care levels, stresses, satisfactions and ways of coping that is gained during assessment. The stress-process model, for example, demonstrates possible foci for intervention at the level of reducing primary or secondary stressors, working to address intrapsychic strain or improving mediators such as coping skills or social support.

Zarit \& Edwards (1999) suggest that following assessment, the clinician needs to consider both the appropriate treatment strategy and the appropriate treatment modality. The main treatment strategies include: the provision of information; assistance with problem-solving to manage stressors; and providing or identifying sources of emotional and practical support. The modalities include one-to-one counselling or therapy; family meetings; and support groups. Although these may sound straightforward, in practice they may be complex because of the intertwined emotional and practical issues involved. For example, a person who attributes a relative's repetitive questions to a lack of attention may need information to understand the nature of memory loss but may be emotionally defended against the knowledge that their relative has dementia.

A meta-analysis of research between 1980 and 1990 (Knight et al, 1993) suggested that psychosocial interventions and respite care delivered on an individual basis each produced a moderate beneficial effect on carer distress. Group psychosocial interventions showed a small positive effect and other health and social services had no consistent impact on carer distress. In a review covering 1987 to 1993, Cuijpers \& Nies (1997) examined 
research on similar areas and concluded that individual interventions have significant positive effects; support groups enable people to use services appropriately; and respite care has limited effects. This is a difficult area to research and, on the whole, studies tend to show promising rather than clearcut results.

\section{Conclusions}

Carers play a vital role in supporting family members who need help. The role carries many satisfactions but is also demanding, and levels of stress and distress among carers are high. The needs of carers should therefore be taken into account, high levels of stress or depression identified and intervention or support provided. Where this is not the case, health services may find themselves with two patients to support where previously there was only one, supported by a carer. This has recently been recognised in government policies and guidelines. It is possible to outline a set of risk factors that make carer stress more likely and these can be subsumed within one of a number of models for conceptualising the carer's reactions. Instruments are also available that can supplement interviews to help in assessment. Individual psychosocial interventions to improve carer well-being are promising, although further research is needed.

\section{References}

Audit Commission (2000) Forget Me Not: Mental Health Services for Older People. London: Audit Commission.

Barusch, A. S. \& Spaid, W. M. (1989) Gender differences in caregiving: why do wives report greater burden? Gerontologist, 29, 667-676.

Baumgarten, M., Battists, R. N., Infante-Rivard, C., et al (1992) The psychological and physical health of family members caring for an elderly person with dementia Journal of Clinical Epidemiology, 45, 61-70.

Bedard, M., Pedlar, D., Martin, N. J., et al (2000) Burden in caregivers of cognitively impaired older adults living in the community: methodological issues and determinants. International Psychogeriatrics, 12, 307-332.

Brody, E. M. (1981) 'Women in the middle' and family help to older people. Gerontologist, 21, 471-480.

-, Hoffman, C., Kleban, M., et al (1989) Caregiving daughters and their local siblings: perceptions, strains and interactions. Gerontologist, 29, 529-538.

-, Litvia, S. J., Hoffman, C., et al (1992) Differential effects of daughters' marital status on their patient care experiences. Gerontologist, 32, 58-67.

Brooker, D. (1998) BASOLL - Behavioural Assessment Scale of Later Life. Bicester: Winslow.

Coen, R. D., Swanwick, G. R., O’Boyle, C. A., et al (1997) Behavioural disturbance and other predictors of carer burden in Alzheimer's disease. International Journal of Geriatric Psychiatry, 12, 331-336.

Croog, S. H., Sudilovsky, A., Burleson, J. A., et al (2001) Vulnerability of husband and wife caregivers of Alzheimer disease patients to caregiving stressors. Alzheimer Disease and Associated Disorders, 15, 201-210.

Cuijpers, P. \& Nies, H. (1997) Supporting informal care-givers of demented elderly people: psychosocial interventions and their outcomes. In Care-Giving in Dementia: Research and Applications (eds B. M. L. Meisen \& G. M. M. Jones), pp. 168-177. London: Routledge.

Deimling, G. T., Bass, D. H., Townsend, A. L., et al (1989) Care related stress: a comparison of spouse and adultchild caregivers in shared and separate households. Journal of Aging and Health, 1, 67-82.

Department of Health (1999a) Caring for Carers. A National Strategy for Carers. London: Department of Health.

- (1999b) National Service Framework for Mental Health. Modern Standards and Service Models. London: Department of Health.

- (2001) National Service Framework for Older People. London: Department of Health

Enright, R. B. (1991) Time spent caregiving and help received by spouses and adult children of brain-impaired adults. Gerontologist, 31, 375-383.

Gerritsen, J. C. \& Van der Ende, P. C. (1994) The development of a care-giving burden scale. Age and Ageing, 23, 483-491.

Hall, J. (1990) Towards a psychology of caring. British Journal of Clinical Psychology, 29, 129-144.

Jones, D. A. \& Vetter, N. J. (1984) A survey of those who care for the elderly at home: their problems and their needs. Social Science and Medicine, 19, 511-514.

Kaye, L. W. \& Applegate, J. S. (1990) Men as Caregivers to the Elderly. Lexington, MA: Lexington Books.

Kinney, J. M. \& Stephens, M. A. P. (1989) Caregiving Hassles Scale: assessing the daily hassles of caring for a family member with dementia. Gerontologist, 29, 328-332.

Knight, B. G., Lutsky, S. M. \& Macofsky-Urban, F. (1993) A meta-analytic review of interventions for caregiver distress: recommendations for future research. Gerontologist, 33, $240-248$

Matson, N. (1994) Coping, caring and stress: a study of stroke carers and carers of older confused people. British Journal of Clinical Psychology, 33, 333-344.

MacLennan, W. (1998) Caring for carers. Age and Ageing, 27, 651-652.

Mui, A. (1992) Caregiver strain among Black and White daughter caregivers: a role theory perspective. Gerontologist, 32, 203-212.

Nolan, M. \& Grant, G. (1992) Regular Respite: An Evaluation of a Hospital Rota Bed Scheme for Elderly People. London Age Concern.

-, - \& Keady, J. (1996) Understanding Family Care. Buckingham: Open University Press.

—, Keady, J. \& Grant, G. (1995) CAMI: a basis for assessment and support with family carers. British Journal of Adult/ Elderly Care Nursing, 1, 822-826.

Office of National Statistics, Social Survey Division (1997) Living in Britain: Results from the 1995 General Household Survey. London: Stationery Office.

Pearlin, L. I., Mullan, J. T., Semple, S. J., et al (1990) Caregiving and the stress process: an overview of concepts and their measures, Gerontologist, 30, 583-594.

Pruchno, R. A. \& Resch, N. L. (1989) Husbands and wives as caregivers: antecedents of depression and burden Gerontologist, 29, 159-165,

Reay, A. M. \& Browne, K. D. (2001) Risk factor characteristics in carers who physically abuse or neglect their elderly dependants. Aging and Mental Health, 5, 56-62.

Schultz, R., O'Brien, A. T., Bookwala, J., et al (1995) Psychiatric and physical morbidity effects of dementia caregiving: prevalence, correlates and causes. Gerontologist $35,771-791$.

Spruytte, N., Van Audenhove, C. \& Lammertyn, F. (2001) Predictors of institutionalization of cognitive-impaired elderly cared for by their relatives. International Journal of Geriatric Psychiatry, 16, 1119-1128.

Zarit, S. H. \& Edwards, A. B. (1999) Family caregiving research and clinical intervention. In Psychological Problems of Ageing: Assessment, Treatment and Care (ed. R. T. Woods) pp.153-193. Chichester: John Wiley \& Sons.

-, Reever, K. E. \& Bach-Peterson, J. (1980) Relatives of impaired elderly: correlates of feelings of burden. Gerontologist, 20, 649-655. 
—, Todd, P. A. \& Zarit, J. M. (1986) Subjective burden of husbands and wives as caregivers: a longitudinal study. Gerontologist, 26, 260-266.

\section{Multiple choice questions}

1 In relation to facts and figures about ageing:

a most carers look after a person under 65 years of age

b 10000 school-age carers look after a parent with mental illness

c less than $25 \%$ of carers are themselves aged over 65

d most care is provided by women

e informal care in the UK costs carers almost $£ 3$ billion per year.

2 In relation to the impact of caregiving:

a over one-third of all carers have significant psychological morbidity

b carer stress may cause institutionalisation for the carereceiver

c carers take more prescribed medication than do noncarers

d carers report more daily hassles than do non-carers

e over a half of all carers report some satisfaction with caring.

3 The following factors are associated with poorer well-being of the carer:

a the degree of instrumental care

$\mathrm{b}$ the degree of social support

c being a daughter rather than a spouse

d disturbed or demanding behaviour of the carerecipient

e being a man rather than a woman.

4 In measuring the impact of caring:

a 'subjective burden' comprises the carer's emotional and cognitive reaction

b 'objective burden' consists of the required service inputs

c objective rather than subjective burden should be measured

$\mathrm{d}$ there are scales for assessing the coping styles of carers

e scales can be used to find a focus for intervention.

5 In relation to models and interventions:

a stress-process models can help define areas for intervention

b carers' groups have no impact on caregiver distress

c day centres have been shown to have a positive effect for carers

d respite care has been shown to have a positive effect for carers

e individual psychosocial intervention has been shown to have a positive effect for carers.

\section{MCQ answers}

\begin{tabular}{|c|c|c|c|c|}
\hline & 2 & 3 & 4 & 5 \\
\hline F & a $\mathrm{T}$ & a $F$ & a $\mathrm{T}$ & a $\mathrm{T}$ \\
\hline $\mathrm{T}$ & $\mathrm{b} T$ & $\mathrm{~b} T$ & b F & $b F$ \\
\hline $\mathrm{F}$ & c $\mathrm{T}$ & c $\mathrm{T}$ & c F & \\
\hline $\mathrm{T}$ & d $\mathrm{T}$ & $\mathrm{d} T$ & $\mathrm{~d} T$ & \\
\hline $\mathrm{T}$ & e $\mathrm{T}$ & e F & e $\mathrm{T}$ & e \\
\hline
\end{tabular}

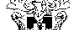
GASKEI

GASKELL

YOU CAN NOW BUY ONLINE

Buy any Gaskell book at www.rcpsych.ac.uk/publications 